\title{
DOI: https://doi.org/10.24297/jap.v15i0.8014
}

\section{Light's Quanta Induce The Measurement Paradox}

\author{
Antonio PUCCINI \\ Neurophysiologist of Health Ministry, Naples - Italy \\ antonio_puccini@libero.it
}

\section{ABSTRACT}

We learn from Quantum Mechanics that the observation of the microscopic world, the measurement (M) of a quantum object, i.e. a particle, inexorably modifies the physical system we wish to examine.

What happens is that with the $M$ it takes place a reduction of the state vectors that is the 'wave function collapse' of the measured particle.

Why does it happen? No one knows.

The enigma of the so-called Measurement Paradox, in our opinion, could be solved if we considered that the light quantum(LQ), as suggested by the Principle of Equivalence Mass-Energy, carries out a dynamic-mass equivalent to its energy. The $L Q$ is indispensable to carry out a $M$.

No M can be carried out without using the quantum of light.

Calculus show that a photon of the optic band hits an electron with a momentum bigger than the mass of the electron itself.

This may explain why the $M$ induces the implosion of the quantum object observed, together with the collapse of its wave function, giving rise to the Measurement Paradox.

Keywords: Light's Quantum(LQ); Measurement(M); Quantum Mechanics(QM); Photon(P); Quantum Object(QO).

\section{Introduction}

1.1 de Broglie associated wave

2.1 Linear Unitary Evolution Phase(U Phase)

2.3 Schrödinger's Wave Function Equation

\section{Discussion}

2.1 Measurement (M) of a Quantum Object

2.2 Wave Function Collapse (WFC)

2.3 WFC and $\mathbf{R}$ Process

2.4 The Link between Measurement and Light Quanta

2.5 The fundamental role played by Planck's Constant 


\subsection{The momentum of Photon}

\subsection{Broken Symmetries and Infinities}

\section{Conclusions}

\section{References}

\section{INTRODUCTION}

The purpose of this paper is to try to lift the veil from the age-old enigma that afflicts Physics: the Measurement's Paradox.

The topic we propose to develop is certainly to be considered among the most fascinating and intriguing of all the Physics: it penetrates and goes into the deepest and hidden meanderings of Quantum Mechanics (QM).

As Feynman reminds us, we would like to emphasize a very important difference between Classical Mechanics and QM : according to QM we must talk about the probability that a particle, or quantum

object(QO), will arrive in a given circumstance. We must imply that "in an experimental arrangement

(or even in the best possible one) it would impossible to predict exactly what would happen. We can only predict the odds! This would mean, if it were true, that Physics has given up on the problem of trying to predict exactly what will happen in a definite circumstance. Yes! Physics has given up. We do not know how to predict what would happen in a given circumstance, and we believe now that it is impossible that the only thing that can be predicted is the probability of different events. It must be recognized that this is a retrenchment in our earlier ideal of understanding nature. It may be a backward step, but no one has seen a way to avoid it[1]".

As it is known, the probability $(\mathrm{P})$ of an event in an ideal experiment is given by the square of the absolute value of a complex number, $\psi$, which is called the probability amplitude[1]:

$$
P=|\psi|^{2}
$$

\section{1 de BROGLIE ASSOCIATED WAVE}

Famously, in 1923 de Broglie suggested, without experimental data, to give particles the same property as waves. He gave each particle a its own wave length depending only on the momentum of the particle itself[2]. Any particle with a momentum( $\mathbf{p})$ "seems to be something periodic, as a wave, with an universal relation between the wave length of the particle, indicated by $\lambda$, and modulus $\mathbf{p}$ of its momentum[3]". In this way we have the formula:

$$
\lambda=\frac{\mathrm{h}}{\mathrm{p}}
$$

(where $h$ is the Planck's constant). This is the value of the wave length $(\lambda)$, according to de Broglie formula, which indicates the nature, also wave-like, of all material particles. Therefore, the old question, wave or particle[4][5][6], can be solved with the QM living to the particles - rather, to QO - a wave function(WF) of their own, indicated with $\Psi(\mathrm{x})$, or simply $\Psi$.

It describes correctly both their wave and particle character.

The WF is a mathematical function which depends on time $(t)$ and on the position $(x)$ of the particle it is referred to. "The function $\Psi(\mathrm{x})$ is usually called the wave function because it more often than not has the form of a complex wave in its variables[1]". Feynman adds: "The WF for a single particle is a 'field', in the sense that it is a 
function of position[1]": $\Psi(\mathrm{x})$. "A consequence of the wave nature of $\mathrm{QM}$ is that each (quantum) state corresponds to a wave, and waves can be superimposed[7]".

In fact, QM equations imply a universal presence of superimposed states.

\subsection{LINEAR UNITARY EVOLUTION PHASE (U PHASE)}

The $\mathrm{WF}(\Psi)$, that is the quantum state of the particle, represents the way in which we can find the particle when it does not interacts, when it is not disturbed, measured, observed. Thus, indicating with $t$ the time, and with $\mathrm{x}^{1}, \ldots . . . \mathrm{x}^{\mathrm{N}}$ the possible positions or space coordinates of the considered particle, we have the formula:

$$
\Psi=\Psi\left(x^{1}, \ldots \ldots . . . x^{N}, t\right)
$$

Hence, before we search the particle, that is before we measure it, the particle is spread throughout the employable space, as if for each point there was associated a precise value of probability density we have to find. According to $\mathrm{QM}$, before the measurement(M) the wave or particle aspects are not at all outlined: the square of the modulus of the $\Psi$, that is $|\Psi|^{2}$, has to be interpreted as a distribution, as the density of probability to find the particle, its quantum state, in one of the several possible positions. In other words, the WF square modulus of a $\mathrm{QO}$ is a $\mathrm{M}$ of the probability (and only the probability) that the $\mathrm{QO}$ is, in a specific moment, in a certain position in the space. QOs do not have defined properties until we observe them, until we make a M.

We can just presume approximately their structure and behaviour, but we have no certitude.

\subsection{SCHRODINGER'S WAVE FUNCTION EQUATION}

Before the $M$, the phase of WF gives to the QO its "wave-like character", since the WF is diffused in the space occupied by the particle the WF is referred to. This condition of the WF, indicated as unitary linear phase $\mathbf{U}$, or $\mathbf{U}$ Process, has been brilliantly described by Schrödinger.

The first difficulty he found, was that the WF was as a function of time.

How to add the difference from the time(t)? Indeed the classical Hamiltonian($(\mathbf{H})$ representing, as we know, the total energy of the examined physical system, is independent by the time.

In the Hamiltonian representation the generalised condition positions $\left(x^{1}, \ldots . . x^{N}\right)$ are associated to the conjugated momenta( $\left(\mathbf{p}_{1}, \ldots . \mathbf{p}_{\mathrm{N}}\right)$, so the momentum(p) of a free particle is given by the velocity $(\mathrm{v})$ of the particle, times its $\operatorname{mass}(\mathrm{m})$ :

$$
\mathbf{p}=m v
$$

Thus, according to the Hamiltonian formalism, aiming to describe the total energy of the physical system we are examining, independently by the time, but by momenta and positions, we have the Hamiltonian function(H):

$$
\mathbf{H}=\mathbf{H}\left(\mathbf{p}_{1}, \ldots . \mathbf{p}_{\mathrm{N}} ; \mathrm{x}^{1}, \ldots . . \mathrm{x}^{\mathrm{N}}\right)
$$

As we know, along with the mathematical formalism of the QM, $\mathbf{p}$ can be identified by a Heaviside differential operator(D):

$$
D=\frac{d}{d x}
$$

In this identification, between $\mathbf{p}$ and $\mathrm{D}$, with the $\mathrm{QM}$ we have the quantum momentum( $\left(\mathbf{p}_{\mathrm{a}}\right)$ : 


$$
\mathbf{p}_{\mathrm{a}}=\mathrm{i} \hbar \frac{\partial}{\partial \mathrm{x}^{\mathrm{a}}}
$$

where $\mathrm{i}$ is the imaginary unit. The new momentum operator $\left(\mathbf{p}_{\mathrm{a}}\right)$, typical of the quantum formalism, substitutes the classical momentum (p) in the Hamiltonian classical function, see equation(5), according to the process known as canonical quantization. The $\mathbf{p}_{a}$ in equation(7) was used by Schrödinger[8] in his equation, occupying all the first member, adding the quantum state $\Psi$ which varies according to the time(t):

$$
i \hbar \frac{\partial \Psi}{\partial \mathrm{t}}=\mathbf{H} \Psi
$$

The second member of the equation(8), or Schrödinger's equation, expresses the energy of the examined system, that is of the $\Psi$. This energy is represented, as in the classical form, by the Hamiltonian $(\mathbf{H})$, but in that case it is a quantum Hamiltonian function, as:

$$
\mathbf{H}=\mathbf{H}\left(i \hbar d / d x^{1}, \ldots . . i \hbar d / d x^{N} ; x^{1}, \ldots . . x^{N}\right)
$$

Thus, as we are all aware, the Schrödinger equation is an equation of temporal evolution indicating how the considered physical system, the particle, represented in its quantum state or WF, can change, develop in time. It expresses the phase of linear evolution of the considered particle called ' $\mathbf{U}$ phase', since it is the process of Unitary evolution, which corresponds to the "Process 2" described by von Neumann[9]. It could say that this $\mathbf{U}$ evolution indicates a particle when it is not troubled but it develops linearly, normally, according to the need of the particle itself and its parameters.

This situation persists in time till we observe it, till we make a $M$, or till it interacts by chance with another particle or physical system[10].

\section{DISCUSSION}

\subsection{MEASUREMENT Of a QUANTUM OBJECT}

Let's examine as in the mathematical formalism of QM a $\mathrm{M}$ of a quantum system must be represented: a 'measurable quantity' of a quantum system is represented by a certain kind of operator $\mathbf{Q}$, called observable. Examples of observables are the 'dynamic variables': i.e. the momentum( $\mathbf{p})$ and the position $(x)$ of the particle we wish to examine.

The theory requires that an observable $\mathbf{Q}$ is represented by a linear operator $\mathbf{L}$, so that its action in Hilbert space(HS) is to make a linear transformation of $\mathbf{H S}$.

A primary request for the quantum observables is that their self-vectors cover the entire HS. That is, the selfvectors of the particle we wish to observe - its quantum superimpositions fluctuating inside the space occupied by the particle itself - must move inside the HS. It is the same as saying that the requirement of QM leads the real space occupied by the particle to coincide with the $\mathbf{H S}$.

According to these considerations, the HS should become a real space, not only hypothetical.

In QM the HS coincides with the phases space of classical physics. "The gravitational Lagrangian of Hilbert, indicated with $S$, consists essentially of the scaled bend(R) divided by the constant $16 \cdot G$ (where $G$ is the gravitational constant), multiplied by $\cdot$ :

$$
S=\frac{1}{\int_{v}(L-R) \varepsilon 16 \pi G}
$$

where - represents the quantity normally expressed as: 


$$
\cdot=d x^{\circ} \wedge d x^{1} \wedge d x^{2} \wedge d x^{3} \sqrt{ }\left(-\operatorname{det} \mathbf{g}_{\mathbf{i} \mathbf{j}}\right)
$$

$\mathbf{L}$ is the Linear Operator, which must be considered as space-time density, which means that the invariant entity is the four-dimensional $\mathbf{L}$ - . The integral of the action will be:

$$
\mathbf{S}=\int_{\mathrm{U}} \mathbf{L} \varepsilon
$$

where $\mathrm{u}$ indicates the four-dimensional (complete) volume of space-time[3]". All of this should occur in the HS that, again, should correspond with volume of space occupied by a particle till it is not troubled, observed, measured: i.e. during the time, the phase in which the particle is indeterminate, not localized: the so-called $\mathbf{U}$ Phase.

According to the rules of $\mathrm{QM}$ the result of a $\mathrm{M}$, related to an operator $\mathbf{Q}$ is always one of the two self-states: this is the jump of the quantum state, or Wave Function Collapse(WFC), which occurs with the Reduction Process(R Process). Penrose adds: "Whatever the state before the $\mathbf{M}$, it jumps in one of the $\mathbf{Q}$ self-states, as soon as the state (that is the particles in exam) is measured (along with the $\mathbf{R}$ Process). After the $M$ the state gets a definite value for the observable $\mathbf{Q}$, precisely the self- value $\mathbf{q}$. If the $M$ is repeated, the second $M$ will give the same self-value, that is the same result we got with the first $M[3]^{\prime \prime}$. When the observable $\mathbf{Q}$ is measured on the state $\mid \Psi>$, the rule is that the probability tells us that the state jumps from $\mid \Psi>$ to one of the $\mathbf{Q}$ selfstates: $\mid \cdot>$.

The jump of the WF, or WFC, induced by any kind of $M$, is represented as follows:

$$
|<\Psi| \cdot>\left.\right|^{2}
$$

This is not true, of course, for the macroscopic world.

Therefore, the $\mathrm{M}$ leads to the collapse of WF(WFC) of the observed particle, working in the HS relative to the same particle. However, along with Penrose[3] the WFC, induced by $M$, could represent a real (not only hypothetical) event occurring completely in the reality, so that also the space in which the collapse happens could be probably a real space, not imaginary: objective WFC. And which is the space where the WFC occurs? It is of course the volume of space occupied by the particle before $M$, the space where the superimpositions of quantum states of the observed particle move. And this space could correspond to the HS.

Miller states "According to QM the falling electron can be in any position, since its WF is diffused throughout the space (the ball, instead, is localised since the beginning). It doesn't have any sense to wonder where the electron is, until a $\mathrm{M}$ is carried out, i.e. taking a picture of it: in this case we need to light it up, at least with a light's quantum(LQ), which becomes part of the $M$ system. The interaction of the single LQ with the electron, localises it in that moment[11]", at the same time we have induced a particular phenomenon of the QM : the WFC.

\subsection{WAVE FUNCTION COLLAPSE (WFC)}

The contact of a single LQ with the electron in exam can collapse its quantum states, its WF.

Well, the interaction between the M's system (that is also a single light quantum) and the examined physical system (the electron) induces the $\mathbf{R}$ Process: that is the Reduction of the electron WF (which was diffuse, till a moment before the $\mathrm{M}$ ), so now it tends to converge to a certain, well defined, region of the space. That is "among all the possible positions which the electron WF can occupy, as a diffused wave in all the space, the M process chooses one[11]".

According to QM, before the $\mathrm{M}$, the particle may be represented by a combination of quantum states more or less superimposed. However it is thought that the $M$ itself makes it pass to a particular state. Thus, if we 
consider that an electron is localized in this or that point, the QM tells us that it can accumulate the 2 possibilities, the 2 possible states, and become the sum of an electron which is in this or that point: with the opportunity then to pass through 2 close splits in the same time, until we don't observe it[12].

What is this happening? What kind of mechanism can be concealed behind the M? No one knows. Miller adds "both Schrödinger equation and the other QM fundamental equations remain mute![11]". However, what seems important is that "the WF does not evolve along with Schrodinger equation, after the M[13]".

\subsection{WFC and R PROCESS}

As we know the phase called Reduction Process(or $\mathbf{R}$ Process), which corresponds to the "Process 1" described by von Neumann[9], lasts just a very short moment (fractions of second), as the $M$ effect ends the previous phase is resumed (present knowledge does not clarify why the WFC is so short). According to QM we will never be able to have information about the aspect and the property of a $\mathrm{QO}$, until it is observed. It is thought that before the $M$ the electron could be found potentially in one of the several points of its wave volume, each corresponding to a probability amplitude, to a probability density. It really seems that when the electron (or another particle) is not disturbed, that is no $\mathrm{M}$ is carried out, it stays in its natural state: it lives as a QO.

Thus it occupies a volume, it is spread in the space which is allowed to it (it is delocalized), and it is represented by superimposed quantum state: it tend to behave as a wave.

This is the phase of linear evolution $\mathbf{U}$, described by Schrödinger equation.

On the contrary, with the M the collapse of the WF takes place, so now our particle will be detectable in a precise point, and at the same time the other probability amplitude will disappear. In fact, the WFC is also called Amplitudes Reduction.

What happened is that with the $M$, with the light (necessary to see the particle), it took place a Reduction of the state vectors ( $\mathbf{R}$ Process), that is the WFC of the measured electron.

Therefore, with the $M$ the state of the particles jumps in a localised state: with the $M$ the quantum state of the particle is a self-vector of the position operator $x$. Before the $M$, probably the particle was scattered in a likewave way throughout the space which could be occupied (self-state of the momentum operator $\mathbf{p}$ ). When the electron's WF collapses, it is delimited in a specific point: the particle is localized, its position is detected.

The electron will now show completely as a particle, it is in fact observed in its corpuscular aspect.

A corpuscle is, indeed, something concentrated in a precise point of the space.

In short, the $M$ induces the collapse of the WF particle we want to examine, so it will pass from a wave behaviour to a corpuscular aspect[14].

Penrose says: "It is clear that the WF is something more real than a simple probability wave. Schrödinger equation gives us this entity (both charged and non-charged), a precise evolution in time, an evolution which depends critically on how the phase changes from a point to another. Moreover in QM wave equation energy $(E)$ is expressed by differential operators [3]":

$$
E=i \hbar \frac{\partial}{\partial t}
$$

Physicists wondered what was the role of the observer in the $M$ process of a physical system. Does the chance have a role, or it doesn't, in determining the results of the M? According to Bohr we cannot talk about a particle without taking in account the interaction we, observers, can have with it (in contrast with classical physics). Bohr writes: "The finite interaction between object and measuring agencies conditioned by the very existence of the 
quantum of action entails -because of the impossibility of controlling the reaction of the object on the measuring instruments if these are to serve their purpose- the necessity of a final renunciation of the classical ideal of causality and a radical revision of our attitude towards the problem of physical reality[15]". That is, a physical theory can describe physical phenomenon only if it includes an experimental content, the observation, the $M$, which make these phenomena show (though there are modified). Prigogine replies: "The cosmic microwave background radiation, distributed in the cosmos at $3^{\circ}$ Kelvin, is witness to the beginning of the universe. But the idea that such radiation would be the result of $M$ is absurd: in fact, who could or should measure it? It is therefore necessary in QM to have an intrinsic mechanism that leads to the observed statistical aspects: this mechanism is precisely instability, chaos [16]".

\subsection{The LINK between MEASUREMENT and LIGHT QUANTA (LQ)}

This is the crucial point: the use of the light results in a modification of the quantum state of the particle observed, since it undergoes, under the action of the electromagnetic radiation(EMR), the jump, the collapse of its WF(WFC), thus the particle, the QO, that used to behave as a wave will now appear as a corpuscle.

What is particularly relevant is that to carry out a $\mathrm{M}$, to observe anything in the Universe, any macroscopic object or particle, it is necessary to use a LQ, i.e. a photon $(P)$ having a wave length $(\lambda)$ shorter or equal to the diameter of the object to be observed. In this way the EMR hits the object and, bouncing back partially towards us will give us the information about the object examined.

On the contrary, if the wave length of the EMR is longer than the diameter of the particle or object to examine (i.e. a radio wave), it will go around the object, jump it, and will not show it to us. In the same way since EMR will not hit the object the WFC will not take place.

Hence, the smaller the object or particle to be examined, the smaller has to be the wave length of the EMR used, thus bigger its energy.

Therefore, if we want to detect, observe, measure an electron, we need to light it, we need to point on it an EMR with a short $\lambda$. However in this case we hit it so deviate and modify its trajectory.

Indeed, the QM teaches us that the observation of the microscopic world, the $\mathrm{M}$, modify the physical system we want to examine.

According to our opinion, it seems that the main character in this enigma (the Measurement's Paradox) is the EMR. Why?

The main reason is that in order to observe, to see, or make a $M$, we always need to use the EMR.

It is the only physical mean which allows us to detect a particle, analyse and study the physical system we are interested in[17]. Only using the light quanta we can acquire the information about the state and the property of the objects of the subatomic world.

No M can be made without using the EMR. Without the EMR we wouldn't be able to observe the world: both at a macroscopic and a microscopic level.

The $\mathbf{L Q}$ is the wire which links the observer to the physical system to be observed.

This wire allows us to get the $M$ of the particle we are interested in.

Without this wire we wouldn't have any information of the world, which would appear dark and unknown, and would never be able to observe it, to measure it. 


\subsection{The FUNDAMENTAL ROLE PLAYED by PLANCK's CONSTANT}

Let's analyse the nature of such a radiation. It carries a large number of LQ, or photons (Ps), second after second. The energetic values of each $P$, without considering its oscillating frequency, corresponds to the Planck's constant, which is just an energetic value, corresponding to $6.626 \cdot 10^{-27}[\mathrm{erg} \cdot \mathrm{sec}]$. Barrow says: "The non-null value of the Planck constant $(h)$ is important for the stability of matter. In the impacts between the atoms and the EMR, the value of $h$ is large enough to take a rather strong 'stroke' to push the electrons to the immediately higher permissible level. As it is known, $\mathrm{h}$ identifies with Planck 'grain, with the quantum of light, that is with $\mathrm{P}$. And yet, a massless $\mathrm{P}$ is capable of inferring such a stroke, besides giving "stability to matter[18]". Unless the $P$ is not so massless.

The $P$, of course, goes with the speed of light, this value(c) is know too, it is $299792.458( \pm 0.4) \mathrm{Km} / \mathrm{sec}[19]$. Let's now consider the equation related to the Principle of Equivalence Mass-Energy

(MEEP):

$$
E=m c^{2}
$$

That's how Einstein commented upon his MEEP: "The value of the considered mass refers to the value of an inertial mass[20]". Let's apply Eq.(15) to the P, keeping in mind that one of the three parameters is well known, that is $c$, the speed of the $\mathrm{P}$ in the vacuum. The $2^{\circ}$ parameter is the Energy of the $\mathrm{P}$ which, as described first by Planck[21][22] and later by Einstein[23], is expressed

by the formula:

$$
E=h \cdot v
$$

where $h$ is Planck's constant and $v$ its oscillation frequency. Here things get more complicated since the equation(16) expresses the energetic value of a single LQ (or P) in motion, that is at the highest speed, oscillating a number of times per second, depending on the EMR band to which the quantum of light is associated. The equation(15), instead, represents the value of an inertial mass, just because it is involved the MEEP, it will express an inertial energy, as to say a minimal energy, or Zeta Point Energy(ZPE)[24] of the particle we are considering.

On the other hand, still for the MEEP, to an "energetic" particle, carrying energy, forces etc., should correspond a mass equivalent to the energy carried, divided $c^{2}[25]$. Since there is no zero energy, for the ZPE, there should not be any particle carrying energy, with a zero mass. At this regard, Feynman adds: "energy and mass differ just for a $c^{2}$ factor, which is merely a question of units, so we can say energy is the mass. Instead of having to write the $c^{2}$, we put $\mathbf{E}=\mathbf{m}[26]^{\prime \prime}$. This is another authoritative confirmation of our concepts. Hawking writes: "According to Einstein's equation $\left(E=m c^{2}\right)$, the energy is proportional to the mass[27]" and according to Relativity itself to every form of energy corresponds a mass. Thus to a very small energy, as in the case of $P$, corresponds a very small mass, however $\neq 0$ [28]. In short, it is crucial keeping in mind that the value of the density of mass energy carried out by $h$, by the Planck's grane, although infinitesimal (and without considering its number of oscillations per second) will always $b e \neq 0$ !

Einstein writes to his friend Conrad Habicht: "It has come to my mind a consequence of the study of Electrodynamics. The Principle of Relativity, in association with Maxwell fundamental equations, requires that the mass is a direct measure of the energy contained in a body; the light carries a mass[20]". Galison adds:" Einstein was unsatisfied: he was not satisfied of the analyses of the light. Einstein stated that to any kind of energy is associated a mass[20]".

Thus, according to Einstein there should be a mass associated to the light quantum.

Galison continues: "Planck stated that also the transfer of heat adds a mass[20]". 
What is heat made of? We know,it is made of EMR, that is Ps. Thus, according to Planck, a transfer of radiation, of Ps, from $A$ to $B$ will cause an increase in the mass of $B$. "It seemed that a hot pot was heavier than a cold one, although exactly the same size. It was a new idea: in Newtonian physics there was nothing suggesting a variation in mass as a consequence of the energy[20]".

Thus wherever there is a body, or particle, having energy, there should be in a way (visible or hidden, concealed) a certain mass too, and vice versa: this is what comes from Eq.(15). Einstein adds that based on the calculations of its article containing precisely the $E=m c^{2}[29]$, it emerges that a body that emits $E M R$ necessarily loses mass.

Klein says: "Einstein attributes to this result a universal value, claiming that mass of a body represents a measure of its energy content. Consequently, if this body loses energy, under any form, it also loses mass! The mass, contained within a body, now measures its energy content. Each body having a mass, equally has a 'mass energy'(i.e. energy of mass). Even at rest, a body having mass contains energy[30]" and vice versa.

Thus, in the case of a $\mathrm{P}$ at the inertial state, that is when it interacts with another particle, so it stops running, at least for that infinitesimal moment it will oscillate much less. We will never be able to know with accuracy how much an interacting $P$ can oscillate, that is what could be the number of oscillations[c/s] in that moment. Let's indicate this unknown value with $10^{\mathrm{n}}[\mathrm{c} / \mathrm{s}]$, which is an uncertainty factor. The $\mathrm{P}$ stops running when hitting another particle, as it happens during a $\mathrm{M}$, so it will not oscillate as when it was running, though it never stops running completely: it is the Heisemberg Uncertainty Principle(HUP) to deny it, since in this case we would know simultaneously the position and the momentum of the particle[31][32]. Thus also in the inertial state the oscillating frequency $(v)$ of the $P$ can never be 0 , but always $\geq 1 / s$, that is $\geq$ one oscillation per second (if not even $1 / 2$ oscillation per s., or a fraction of its). Thus, if we want to consider the Energy of the $P$ in its inertial state, indicated with $\mathrm{E}_{\mathrm{o}}$, we should have:

$$
\mathrm{E}_{\mathrm{o}}=\mathrm{h} \cdot \mathrm{v}=\mathrm{h} \cdot 10^{\mathrm{n}}[\mathrm{c} / \mathrm{s}]
$$

that is:

$$
\mathrm{E}_{\mathrm{o}}=6.626 \cdot 10^{-27}[\mathrm{erg} \cdot \mathrm{s}] \cdot 10^{\mathrm{n}}[\mathrm{c} / \mathrm{s}]
$$

hence:

$$
\mathrm{E}_{\mathrm{o}}=6.626 \cdot 10^{-27+\mathrm{n}}[\mathrm{erg}]
$$

This should be the Energy value of a $P$ at an inertial state. We may say its minimal energy value; as we can see this value is not easy to determine, rather, it is undetermined, as stated by the QM.

As the erg value is expressed in $\left[\mathrm{g} \cdot \mathrm{cm} / \mathrm{s}^{2} \cdot \mathrm{cm}\right]$, that is in $\left[\mathrm{g} \cdot \mathrm{cm}^{2} / \mathrm{s}^{2}\right]$, we have:

$$
E_{o}=6.626 \cdot 10^{-27+\mathrm{n}}\left[\mathrm{g} \cdot \mathrm{cm}^{2} / \mathrm{s}^{2}\right]
$$

In this way we can have information, with a certain approximation, about the $2^{\text {nd }}$ parameter of equation(15), referred to the $P$. Hence we can easily have the $3^{\text {rd }}$ parameter, the equivalent rest-mass or equivalent inertial $\operatorname{mass}\left(m_{\circ}\right)$ of the $P$ :

$$
\mathrm{m}_{\mathrm{o}}=\frac{\mathrm{E}_{\mathrm{o}}}{\mathrm{c}^{2}}=\frac{6.626 \cdot 10^{-27+\mathrm{n}}\left[\mathrm{g} \cdot \frac{\mathrm{cm}^{2}}{\left.\mathrm{~s}^{2}\right]}\right.}{\left(2.9979 \cdot 10^{10}\right)^{2}[\mathrm{~cm} / \mathrm{s}]^{2}}
$$

Let us calculate this value following the cgs system: 


$$
\mathrm{m}_{\mathrm{O}}=\frac{6.626 \cdot 10^{-27+\mathrm{n}}}{(2.9979)^{2}} \cdot 10^{-20} \cdot \frac{\left[\mathrm{g} \cdot \frac{\mathrm{cm}^{2}}{\mathrm{~s}^{2}}\right]}{\frac{\mathrm{cm}^{2}}{\mathrm{~s}^{2}}}
$$

and we have:

$$
\begin{aligned}
& \mathrm{m}_{\mathrm{O}}=\frac{6.626}{(2.9979)^{2}} \cdot 10^{-27-20+\mathrm{n}} \cdot\left[\mathrm{g} \cdot \frac{\mathrm{cm}^{2}}{\mathrm{~s}^{2}}\right] \cdot \frac{\mathrm{s}^{2}}{\mathrm{~cm}^{2}} \\
& \mathrm{~m}_{\mathrm{O}}=\frac{6.626}{(2.9979)^{2}} \cdot 10^{-47+\mathrm{n}}[\mathrm{g}]
\end{aligned}
$$

that is:

$$
m_{0}=7.372 \cdot 10^{-48+n}[g]
$$

What we get is that the inertial mass energy of the $P$ corresponds to $10^{-48+n}$ grams. Thus, if the value of $n$ was $10^{0}$, that is one oscillation per second, $m_{0}$ would be $10^{-48}[\mathrm{~g}]$. Whereas if $\mathrm{n}$ was $10^{3}$ oscillation per second, we would have $\mathrm{m}_{\mathrm{o}}=10^{-45}[\mathrm{~g}]$. Of course in all cases it is an extremely small value, but it is $\neq 0$, according to $Q M$ : Zero Point Motion[24]. Besides, as we know, one of characteristics of the $P$ is to travel most of the time, so it also gets a momentum(p).

\subsection{The MOMENTUM of PHOTON}

Feynman says: "Each P has an energy and a momentum(p)[26]".

Penrose states: "According to QM, the key concept concerns the momentum (p): in all shocks, in all interactions between QO, it is always preserved. Momentum must move somewhere: it can not simply disappear, as it is preserved. The same happens for energy[3]". Fermi writes: "The P too, as other particles, is a corpuscle, a LQ, and has a its own momentum(p), through which transfers all its energy to the hit particle[33]".

Feynman adds: "The momentum, as a mechanical quantity, is difficult to hide. Nevertheless, momentum can be hidden -in the electro-magnetic(EM) Field, for example. This case is another effect of relativity[1]". It's like saying that momentum carries, albeit hidden, a dynamic-mass.

In short, the P cannot be considered massless. Its mass is simply, to say it with Feynman: "hidden". Penrose chases: "In a conference held in Japan in 1922, Einstein said: 'If a person falls freely he will not feel his own weight'. In fact, when you are in free fall (like when you launch from a plane, before you open the parachute) you have the impression that the earth gravity interaction(GI) is suspended: the Earth's gravitational field seems to have disappeared. Where's the GI? Actually the GI has not vanished, it is hidden[13]". Well, in these circumstances, we seem to be able to see a significant behavioral analogy between EM Field and gravitational field. That is, it is as if in both of them something disappeared, temporarily concealed, hidden, during the event: 1) the dynamic-mass, transported by the momentum of the P (in the EM Field); 2) the GI (in the gravitational field). The momentum(p) is also represented in the de Broglie's formula(equation 2):

$$
\mathbf{p}=\frac{\mathrm{h}}{\lambda}
$$


where $\lambda$ is the wave length of the considered $P$ (or other particles). As Weinberg reminds us, the mean wave length of a $P$ in the optical band corresponds to $\approx 5 \cdot 10^{-5}[\mathrm{~cm}][34]$ and its $\mathbf{p}$ is:

$$
\begin{aligned}
& \mathbf{p}=\frac{6.626 \cdot 10^{-27}[\mathrm{erg} \cdot \mathrm{s}]}{5 \cdot 10^{-5}[\mathrm{~cm}]} \\
& \mathbf{P}=\frac{6.626 \cdot 10^{-27}\left[\mathrm{~g} \cdot \frac{\mathrm{cm}^{2}}{\mathrm{~s}}\right]}{5 \cdot 10^{-5}[\mathrm{~cm}]} \\
& \mathbf{p}=1.325 \cdot 10^{-22}\left[\mathrm{~g} \cdot \frac{\mathrm{cm}}{\mathrm{s}}\right]
\end{aligned}
$$

Let's see how heavy an electron is: its mass corresponds to $9 \cdot 1 \cdot 10^{-28} \mathrm{~g}$, comparing these values, emerges that a running $P$ is heavier than an electron. Thus, when we make a $M$, when we try to see and study an electron, and we shoot against it even a single $\mathrm{P}$ (the minimum quantity to be able to see it), what happens is that the electron is hit by a corpuscle with a dynamic-mass bigger than its, most likely succumbing under its mechanical effect, under such a shot, thus it collapses.

Every time a $\mathrm{M}$ is carried out (always using the EMR), the observed particle undergoes a probabilistic reduction of the state vector, indicated as Reduction Process, or $\mathbf{R}$ Process. With the $\mathbf{R}$ Process the state vector, represented by $\mid \Psi>$, jumps to another stated vector, let's say $|\phi\rangle$, which represents one out of two or more orthogonal alternative possibilities: the other can be $|q>| X$,$\rangle , etc..., which depend on the kind of$ observation, the kind of $\mathrm{M}$ carried out.

Thus, with the $M$ we move immediately from the phase $\mathbf{U}$ to $\mathbf{R}$, and the jump of the quantum state is induced, known as WFC. All related to the light's quanta, to the EMR[35].

\subsection{BROKEN SYMMETRIES and INFINITIES}

As you will be aware, in agreement with the MEEP, to an "energetic" particle, carrying energy, forces etc., should correspond a mass equivalent to the energy carried, divided $c^{2}$. Since there is no zero energy for the ZPE, as Chandrasekhar reminds us[36], there should not be any particle carrying energy, with a zero mass(MEEP). Then, it may be incongruous to say that a particle with energy does not have an equivalent mass, it does not "conceal", at least, a mass. It is Einstein's equation to show that this particle has a mass, otherwise the equation would be null, the result would be zero. Thus, there should not be real particles, having any energy, with a zero mass. If there are, they should "subtend" a tiny mass, a Zero Point Mass[24]. Hence to a very small energy, as in the case of $P$, corresponds a very small mass, however $\neq 0[28]$.

Therefore, we think that the base concept of the gauge theories: 'the mass breaks the symmetry' is not applicable to the Planck constant. No! Planck's constant - $6.626 \cdot 10^{-27}[\mathrm{erg} \cdot \mathrm{sec}]$ - is a real value, ineradicable: represented by an intrinsic value, it expresses the value of the density of energy-(equivalent mass) of the Planck Quantum. Thus, reduce this value to zero, in order to correct the divergences and infinities emerging from the equations of the Perturbation Calculus, would totally cancel the very existence of LQ and, consequently, also the energy of light: we would have a world everywhere dark and totally devoid of power! No, it is not possible.

However, in our opinion two ways could provide a solution: 1) Correct the infinities, without Renormalization; or: 2) Assume an extension of the Higgs Mechanism. Let's analyze the problem.

1) As known, Oppenheimer demonstrated that at the origin of the infinities there was the term expressing the interaction between the electronic current and the EM Field produced by the electron. Oppenheimer writes: "It is further shown that it is impossible on the present theory to eliminate the interaction of a charge with its 
own field, and that the theory leads to false predictions when it is applied to compute the energy levels and the frequency of the absorption and emission lines of an atom[37]". That is, the self-interaction of the electron, considering the processes in which the electron emits and resets a $\mathrm{P}$, causes an infinite shift (with quadratic divergence)[38]. On the contrary, in order to eliminate the infinites, in our opinion it would be necessary to replace a massless $P$, with the value of the Planck constant $(h)$, or LQ, equal to $7.372 \cdot 10^{-48}[\mathrm{~g}]$, multiplied by the value of the frequency of the considered $P$ : see equation(25).

Weisskopf recalculates the electron self-energy, however, one always gets an infinite, with logarithmic divergence [39]:

$$
\mathrm{E}=\frac{3 \mathrm{e}^{2} \mathrm{mc} \mathrm{c}^{2}}{\mathrm{hc}} \log \left[\frac{\mathrm{h}}{\mathrm{mca}}+\sqrt{1+\left(\frac{\mathrm{h}}{\mathrm{mca}}\right)^{2}}\right]
$$

where $E$ is the electron self-energy, $m$ its mass and a its ray, considered as a point (thus 0 ). In equation (30) the null value of a appears twice in the denominators: we shouldn't marvel at the infinities! Obviously this occurs because in the equations a point value for the radius of the electron (a) is introduced, thus a $\rightarrow 0$ (which is as to give the value $a=0$ ).

Thus, the calculation results in an infinite shift: for $\mathrm{a} \rightarrow 0$ diverges as $1 / \mathrm{a}^{2}$.

Clearly, being massive particles. the electrons can in no way occupy a void or point volume of space, that is, equal to 0 .

To this purpose, Feynman comforts us: "Maybe the idea that two points may be infinitely close is incorrect, it is false the assumption that geometry will continue to be invariably unchanged[40]". He adds: "But if instead of including all the possible points of interaction until a 0 distance, the calculation is cut off when the distance between the points is very small, there exist defined values of the mass of the electron and of the its charge, such that the calculated mass coincides with the value of the mass of the electron measured experimentally, and the calculated charge coincides with the experimental value of the electric charge of the electron[40]".

In short, in our opinion, the removal of the infinites emerging from the perturbative study of QED and other Quantum Field Theories(QFT), can be obtained in two ways. 1) replacing in the equations of such theories the value of 0 of a massless $P$, with the real energy value of the Planck constant, as represented by equation (25). 2) replacing in the equations of the QFT the point value attributed to the radius of the electron, therefore $\rightarrow$ 0 , with the real value of its radius which, according to de Broglie, "presents a wave lenght of the order of $10^{-8}$ $10^{-9} \mathrm{~cm}$, i.e. as a $X$ ray[41]".

2) On the other hand, it is not possible to exclude a priori that another Higgs Boson(HB)[42][43], other than that found at CERN, may possibly allow the $P$ to gain mass, according to an Higgs Mechanism analogous to that proposed by Standard Model.

Namely, the HF is a weak isospin doublet, that is, it has two components, which appear indistinguishable from Weak Interaction(WI)[44][45]. These two components have respectively electric charge +1 and 0 : the HB is associated to the real part of the neutral field, which, by coupling with itself, i.e. with its own field (HF), acquires mass. On the contrary the $\mathrm{P}$, considered insensitive to $\mathrm{WI}$, is massless[44]. However, Penrose chases: "Already at $\leq 10^{16}{ }^{\circ} \mathrm{K}$, at $\approx 10^{-12}$ seconds after the Big Bang, the photon $(\mathrm{P})$ and $\mathrm{W}$ and $\mathrm{Z}$ bosons were frozen by the so-called spontaneous symmetry breaking (SSB) process, so only $\mathrm{P}$ remains massless while the others gain mass. Maybe it is the HB to give masse to these particles, as well as to itself and quarks. And how? Really great and ingenious ideas[3]". Penrose adds: "I question the reality of SSB! There are various difficulties in this idea of SSB[3]".

Feynman, who for Renormalization received the Nobel Prize, almost 40 years later writes: "This compass game, made with the value of the electron rest mass and the value of its 'charge' (i.e. its amplitude of 
interaction with Ps), is called with a technical language renormalization: a fine name for what remains an absurd process! Having had to resort to such prestigious games made it impossible to prove the internal coherence of QED. It is, in fact, surprising that this coherence is still undemonstrated and personally suspect that renormalization is not a mathematically legitimate process. What is certain is that we do not have a good mathematic basis to formulate QED theory [40]". Passera writes: "in reality, no electric charge is point-like, so the problem of the infinites, considering the radius of the electron $\rightarrow 0$ (for which the energy $\rightarrow \infty$, that is, diverges) is simply a pure mathematical abstraction[46]".

In conclusion, in case the concept of SSB is likely, according to Randall (who hypothesizes more complex Higgs sectors[47]) we could imagine that the photon $(P)$ can also acquire mass, through the other component of the HF.

\section{CONCLUSIONS}

The measurement(M), thus, produces a big changes on the physical properties of the observed particle, of the measured QO, as well as on its morphological configuration.

How do these changes happen? What kind of mechanism can be concealed behind the observation,

behind the $\mathrm{M}$, behind this kind of interactions? What is the secret mechanism which creates the

Measurement Paradox(MP)? No one knows.

We only know that these modifications happen any time we try to see how a physical phenomenon takes place, or when we want to study the behaviour of a particle: to do so we have to carry out a M. Thus, the MP and the WFC take place every time $\mathrm{M}$ is carried out.

Which mean do we use to carry out a M? A LQ with a short wave length.

Thus, it is automatic to link together the four parameters: 1)Light; 2)M; 3)WFC; 4)MP.

In fact the WFC and the MP happen only after a M, and the M cannot be carried out without using the light: it is a conditio sine qua non.

Thus, we can infer that the WF of the observed particle, $\mid \Psi>$, jumps in a different quantum state $(|\phi\rangle)$ when the LQ occurs. Without EMR it would not be possible to have neither the $M$, nor, as a consequence, the WFC and MP.

There is no other explanation. Someone may say: if it is so, how does EMR induces the MP?

Well, we have stated that the Planck's constant, the LQ, is not evanescent, ethereal, inconsistent, but it carries out an intrinsic energy value equal to $6.626 \cdot 10^{-27}[\mathrm{erg} \cdot \mathrm{sec}]$.

Besides, EMR produces a mechanical action: the so called "radiation pressure of Ps[48]". For example, "the solar light gives, on the earth surface, a radiation pressure having a weight of $1 \mathrm{mg}$ per $\mathrm{mt}^{2}$ per second[49]". We know that if a single $P$ hits an electron changes its journey and deviates it from its trajectory. In the same way, we think that the $P$ is able to create the WFC of the hit electron and the mysterious MP.

The mechanical effect carried out by an optic $P$ against an electron, against a $\mathrm{QO}$, is not at all negligible: as shown in equation(29), the electron is hit by a crash force equal to $10^{-22}[\mathrm{~g} \cdot \mathrm{cm} / \mathrm{s}]$, that is 100.000 times bigger than the mass of the electron itself[50]. It is a considerable strike! 
There is no wonder if, after such a strike, the quantum structure of the electron (with its superimpositions of quantum states), and its morphological configuration, undergo a significant modification. It is as if under the hit with the LQ, the electron deformed immediately (thug just for a very short time), as if it shrivelled (as pinched balloon), reducing its quantum states: in this way showing itself as a corpuscle, a localised and observable particle. Just with a single P.

Therefore, the light really hit violently the electron and the atomic particles. Before being hit by EMR, in according to the QM the particle is a mathematical quantity known as a quantum state, or $W F(\mid \Psi>)$, that should contain all the information necessary to describe the considered quantum system. When it exists in this phase (U phase), not disturbed, the particle will not give any information concerning its look and contents.

To this purpose, Prigogine asks himself: "Does a unobserved nature, different from observed nature?[16]". It seems so! In fact, as far as we try to see it, the observed particle immediately change its look, its quantum configuration and its trajectory.

Therefore, with $M$, i.e. under the action of $L Q$, the particle, that is its $W F$, jumps in a particular quantum status ( $\phi$, for example), giving rise to the WFC and, consequently, to the MP.

In closing, according to QM a physical phenomenon occurs if somebody is observing it. Therefore the act itself of observing, measuring a sub-particle, i.e. a QO, induces consequently a physical phenomenon. But in which way we can observe a particle? It is enough a LQ sufficiently energy. To this purpose Feynman said: "To observe electrons, we need a light because the light rebounding on electrons make them visible. Nevertheless the light affects the result because the result of light on is different from that of light off. We can say that the light affects electron behaviour. The electrons are very sensitive. When light is sent on an electron, it makes the electron vibrate so that the electron because of light, behaves in a different manner[26]".

In short, it seems that the light is the keystone to observe a particle, to make a $\mathbf{M}$.

Similarly, only through the EMR we can try to reveal the enigma of MP.

In which way? We explained above, it could be a mechanical effect induced by LQ to play a main role with the $M$ and unravel the mysteries of its Paradox.

In conclusion, we think this is just what happens with the $\mathrm{M}$ : the momentum of $\mathrm{P}$ is transferred entirely to the stroked particle(according to Fermi[33],Feynman[1] and Penrose[3],i.e.), respecting the Momentum Conservation Law.

It should just be the moving of the P's momentum to the particle undergoing a $M$, to make the collapse of its wave function(WFC) and make less enigmatic the Measurement's Paradox.

\section{REFERENCES}

1) Feynman R..P., "The Feynman Lectures on Physics",( 1965 , California Institute of Technology, Volume 3: "Quantum Mechanics"; Zanichelli ed.,Bologna,Vol.3,1-13,14;16-7;21-1; (2001).

2) de Broglie L., "Ondes et quanta", Comtes Rendus, Vol.177, 507-510 (1923).

3) Penrose R.,"The Road to Reality",2004 Roger Penrose; 2005,RCS ed.,Milano,500, 484-490, 540, 499,520,529$533544,641,786$ (2005).

4) Descartes R., "Dioptrique”, Adam-Tannery eds., Paris, 84 (1637). 
5) Newton I., Phylosophical Transactions of the Royal Society,Vol.7, 80, 3075-3087 (1672).

6) Young T.,Phylosophical Transactions of the Royal Society, Vol.92,12-48 (1802).

7) Lloyd S., Programming the Universe. A Quantum Computer Scientist Takes on the Cosmos, ( $) 2006$ Seth Lloyd; Einaudi ed.,Torino,123 (2006).

8) Schrödinger E., Annal. der Physik, 79, 361-376 (1926).

9) von Neumann J., "Mathematical Foundations of Quantum Mechanics", Princeton University Press, New Jersey (1955).

10) Puccini A., "The Momentum of Luminous Photon Can Explain the Mistery of the Scission of The Water Molecule, Fundamental Event in the Clorophyllose Photosynthesis", Proceedings of Progress in Electromagnetics Research Symposium, Moskow, Russia, 353-357, August 19-23 (2012).

11) Miller A.I., in "It Must be Beautiful. Great Equations of Moderne Science"; Granta Publ.,London,2002; il Saggiatore ed.,Milano,186-187 (2005).

12) Puccini A., "About the Interference Induced by Electrons. Why does the Electron Behave like a Wave", Progress in Electromagnetics Research, 58,199-222, 2006.

13) Penrose R., in "It Must be Beautiful. Great Equations of Moderne Science"; Granta Publ., London,2002; il Saggiatore ed.,Milano,293,261 (2005).

14) Puccini A., "Anderson Transition May Be Induced by the Self Collapse of the Electron Wave Function", Proceedings of Progress in Electromagnetics Research Symposium, Moskow, Russia, 348-352, August 19-23 (2012).

15) Bohr, N., "Can Quantum-Mechanical Description of Physical Reality be Considered Complete?", Physical Review,48, 696-702 (1935).

16) Prigogine I., "Le Leggi del Caos", Laterza ed., Roma-Bari,61,59 (1993).

17) Puccini A., "The Momentum of Photon May Explain the Measurement's Paradox in the Subatomic World",Session 1A2, Proceedings of Progress In Electromagnetics Research Symposium, Suzhou, China, 2730, Sept. 12-16 (2011).

18) Barrow J.D.,"The Constants of Nature. From Alfa to Omega",@2002 John D. Barrow, Jonathan Cape 2002; Mondadori Ed.,Milano,293 (2003).

19) Achenbach J., National Geographic, Italian edition, Milano,10,8 (2001).

20) Galison P.,L'equazione del Sestante: $E=m \cdot c^{2}$ in "It Must be Beautiful. Great equations of Moderne Science"; Granta Publ.,London,2002;il Saggiatore ed.,Milano,119 (2005).

21) Planck M., Verhandlungen der Deutschen Physikalischen Geseeshaff,Vol.2, 237 (1900).

22) Planck M., Annalen der Physik,Vol.4,553 (1901).

23) Einstein A., Jahrbuch der Radioaktivitat und Elektronik,Vol.4,443 (1907). 
24) Puccini A., "About the Zero Point Energy, Zero Point Mass, Zero Point Temperature and Zero Point Motion in the Subatomic World and Photonics", Proceedings of Progress in Electro-magnetics Research Symposium, Suzhou, China, 1169-1171, Sept. 12-16 (2011).

25) Puccini A., "The Equivalent Rest-mass of Photon", Proceedings of Progress in Electro-magnetics Research Symposium,Session 2P2, Marrakesh, Morocco,723-725 Mar.20-23 (2011).

26) Hawking, S., "A Brief History of Time",৫1988 by Stephen W. Hawking, Bantan Books ed.; Rizzoli ed.,Milano,124-128,90 (1990).

27) Puccini A., "About the Zero Mass of Photon", Progress in Electromagnetics Research, Vol.55,117-146 (2005).

28) Feynman R..P., "The Feynman Lectures on Physics", $(1965$,California Institute of Technology; Zanichelli ed.,Bologna,Vol.1,17-7,15-15,39-8,34-10,10-12,34-16 (2001).

29) Einstein A., Annalen der Physik,Vol.18,639-641 (1905).

30) Klein E.,"Il etait sept fois la revolution",2005 ed. Flammarion; Cortina ed.,Milano,64-65(2006).

31) Heisemberg W., Zeitschrift fur Physik,Vol.43,172-198 (1927).

32) Puccini A., "Uncertainty Principle and Electromagnetic Waves", Journal of Electromagnetic Waves and Applications,Vol.19, No7, 885-890, 2005.

33) Fermi E. "Argomenti pro e contro l'ipotesi dei quanti di luce", Nuovo Cimento,3,201-206,1926.

34) Weinberg S., "The First Three Minutes. A Modern View of the Origin of the Universe", @1977 by S.Weinberg; Mondadori Ed.(Milano),75 (1977).

35) Penrose R., "The Emperor's New Mind", (c) 1989 Oxford Univerity Press; Rizzoli (RCS) Ed., Milano,322,582,287,285,37,3445 (1997).

36) Chandrasekhar B.S., "Why Things Are the Way They Are", Cambridge University Press,1998; Il Saggiatore ed., Milano,304 (2004).

37) Oppenheimer J.R. ,"Electron and Gravitation.1",Phys.Rev.,1930,35,562 (1930).

38) Peruzzi G., "Richard Feynman. QED: un po' di storia”, LNF, 7 ottobre 2015.

39) Weisskopf V.F., "Über die Selbstenergie des Elektrons",Zeitschrift fur Physik,Volume 89, Issue1-2, 27-39 (1934).

40) Feynman, R. P.,"QED. The Strange Theory of Light and Matter",@1985 Richard P. Feynman; Adelphi ed., Milano, 158,156-160, 175,184-185 (1989).

41) De Broglie M.L., "Notice sur les Travaux Scientifique”, Hermann\&C.,Ed.,Paris,26 (1931).

42) Higgs P.W., "Broken Symmetries and the Masses of Gauge Bosons", Phys.Rev.Lett.13,508-509 (1964).

43) Puccini A., "Higgs Boson Radius of Action", International Journal of Innovation Engineering and Science Research, Volume 2, Issue 6, 43-46 (2018).

44) Corcella G., "Il campo di Higgs", INFN, Scienza Per Tutti, Gennaio (2017). 
45) Puccini A., On the Boson's Range of the Weak Interaction, Journal of Advances in Physics, Volume 14,Issue 3, 5865-5868 (2018).

46) Passera M., "L'Infinito sotto il tappeto", asimmetrie.it INFN Padova, aprile (2016).

47) Randall L., "Knocking on Heaven's Door",@ Lisa Randall 2011,2012; il Saggiatore ed., (Milano), 293,307,XIV (2012).

48) Nat'I Academics Press, The National Academy of Science, USA, "Nuclear Physics"111 (1986).

49) Frova A. "La Fisica sotto il naso", RCS ed.,Milano,131 (2001).

50) Puccini A., "The Momentum of Photon May Explain the Measurement's Paradox in the Subatomic World", Session 1A2, Proceedings of Progress In Electromagnetics Research Symposium, Suzhou, China, 2730, Sept. 12-16 (2011).

51) PucciniA.,"A Mechanical Effect Induced by Electromagnetic Radiation May Explain the Wave Function Collapse of a Quantum Object", Session 2P2,Proceedings of Progress in Electro-magnetics Research Symposium, Marrakesh, Morocco, 726-728, Mar. 20-23 (2011). 